\title{
Nonequilibrium Bond Forces in Single-Molecule Junctions
}

Brand, Jonathan; Leitherer, Susanne; Papior, Nick Rübner; Néel, Nicolas; Lei, Yong; Brandbyge, Mads; Kröger, Jörg

\section{Published in:}

Nano Letters

Link to article, DOI:

10.1021/acs.nanolett.9b02845

Publication date:

2019

Document Version

Peer reviewed version

Link back to DTU Orbit

Citation (APA):

Brand, J., Leitherer, S., Papior, N. R., Néel, N., Lei, Y., Brandbyge, M., \& Kröger, J. (2019). Nonequilibrium Bond Forces in Single-Molecule Junctions. Nano Letters, 19(11), 7845-7851.

https://doi.org/10.1021/acs.nanolett.9b02845

\section{General rights}

Copyright and moral rights for the publications made accessible in the public portal are retained by the authors and/or other copyright owners and it is a condition of accessing publications that users recognise and abide by the legal requirements associated with these rights.

- Users may download and print one copy of any publication from the public portal for the purpose of private study or research.

- You may not further distribute the material or use it for any profit-making activity or commercial gain

- You may freely distribute the URL identifying the publication in the public portal

If you believe that this document breaches copyright please contact us providing details, and we will remove access to the work immediately and investigate your claim 


\title{
Nonequilibrium Bond Forces in Single-Molecule Junctions
}

\author{
Jonathan Brand, ${ }^{\dagger}$ Susanne Leitherer, ${ }^{\ddagger}$ Nick R. Papior, $₫$ Nicolas Néel, ${ }^{*, \dagger}$ Yong \\ Lei $^{\dagger}$ Mads Brandbyge, ${ }^{\ddagger}$ and Jörg Kröger ${ }^{\dagger}$ \\ $\dagger$ Institut für Physik, Technische Universität Ilmenau, D-98693 Ilmenau, Germany \\ $\ddagger$ Center for Nanostructured Graphene, Department of Physics, Technical University of \\ Denmark, DK-2800 Kongens Lyngby, Denmark \\ IDepartment of Applied Mathematics and Computer Science, Technical University of \\ Denmark, DK-2800 Kongens Lyngby, Denmark \\ E-mail: nicolas.neel@tu-ilmenau.de,fax:+49-3677-693602
}




\begin{abstract}
Passing a current across two touching $\mathrm{C}_{60}$ molecules imposes a nonequilibrium population of bonding and antibonding molecular orbitals, which changes the equilibrium bond character and strength. A current-induced bond force therefore contributes to the total force at chemical-bond distances. The combination of first-principles calculations with scanning probe experiments exploring currents and forces in a wide $\mathrm{C}_{60}-\mathrm{C}_{60}$ distance range consistently evidences the presence of current-induced attraction that occurs when the two molecules are on the verge of forming a chemical bond. The unique opportunity to arrange matter at the atomic scale with the atomic force and scanning tunneling microscope tip has enabled closely matching molecular junctions in theory and experiment. The findings consequently represent the first report of current-induced bond forces at the single-molecule level and further elucidate the intimate relation between charge transport and force. The results are relevant to molecular electronics and chemical reactions in the presence of a current.
\end{abstract}

Keywords: density functional theory, nonequilibrium Green's function, atomic force microscopy, scanning tunneling microscopy, single-molecule junction, $\mathrm{C}_{60}$

The measurement of forces at the atomic scale with piconewton resolution using an atomic force microscope (AFM) has tremendously advanced physics research in the last decade. Seminal works discovered the spin texture of magnetic surfaces, ${ }^{1,2}$ explored forces to move single atoms, ${ }^{3}$ and unraveled the atomic skeleton of molecules. ${ }^{4,5}$ Conventionally, these forces are described as a superposition of long-range van der Waals and electrostatic attraction together with short-range Pauli repulsion, which have to be supplemented in cases of magnetic materials, ${ }^{1}$ ionic crystals or polar molecules, ${ }^{6,7}$ and surfaces with low conductivity. $^{8}$

Forces induced by current flow were previously discussed in the context of electromigration, ${ }^{9-12} i$.e., the transport of matter through conductors in the presence of a dc current. More recently, the impact of a current across atomic wires ${ }^{13-15}$ and single molecules ${ }^{16-24}$ was 
scrutinized, with emphasis on theoretical modeling. ${ }^{13-17,19,21-24}$ The experimental works report local-heating effects of the current without imaging the individual molecule attached to the electrodes. ${ }^{18}$ While the rotational state of single-molecule electric motors was controlled by local injection of an electric current and imaged with a scanning tunneling microscope (STM), force measurements were not involved. ${ }^{20}$

Experiments exploring the interplay between forces and currents at the single-atom or single-molecule level with precise characterization of the junction are very scarce. For atomic contacts it was shown by using an AFM and an STM that short-range forces and junction conductance exhibit an exponential behavior with characteristic decay lengths in the tunneling range ${ }^{25}$ and are thus correlated. In another experimental work the influence of the tunneling current on the force was unraveled for samples with low conductivity. ${ }^{8}$ Both reports $^{8,25}$ did not consider bond forces.

Here, we go beyond previous reports by combining state-of-the-art density functional theory (DFT) and nonequilibrium Green's function (NEGF) calculations ${ }^{26-31}$ with AFM-STM experiments on artificially fabricated molecular junctions that closely match the model setup. The calculations unveil a current-induced attraction between the $\mathrm{C}_{60}$ molecules that reflects the nonequilibrium population of bonding and antibonding molecular orbitals. The resulting nonequilibrium bond force is dominant for elevated junction conductance in the Pauli repulsion distance range and exhibits a nearly parabolic evolution with the bias voltage. Force spectroscopy in the noncontact frequency modulation mode of AFM with a $\mathrm{C}_{60}$-terminated tip and $\mathrm{C}_{60}$ molecules adsorbed on $\mathrm{Cu}(111)$ ranging from far tunneling to chemical-bond distances is in accordance with the theoretical predictions. After removing van der Waals, electrostatic and Pauli force contributions to the measured total force the residual force component exhibits all characteristics of the calculated current-induced force.

Figure 1a shows the setup used in the calculations. Top $(t)$ and bottom $(b)$ electrodes are represented by $\mathrm{Cu}(111)$ slabs each. Three $\mathrm{Cu}$ atoms arranged in a triangle at $\mathrm{Cu}(111)$ nearest-neighbor sites are attached to the top electrode. $\mathrm{C}_{60}$ molecules are adsorbed to $t$ 

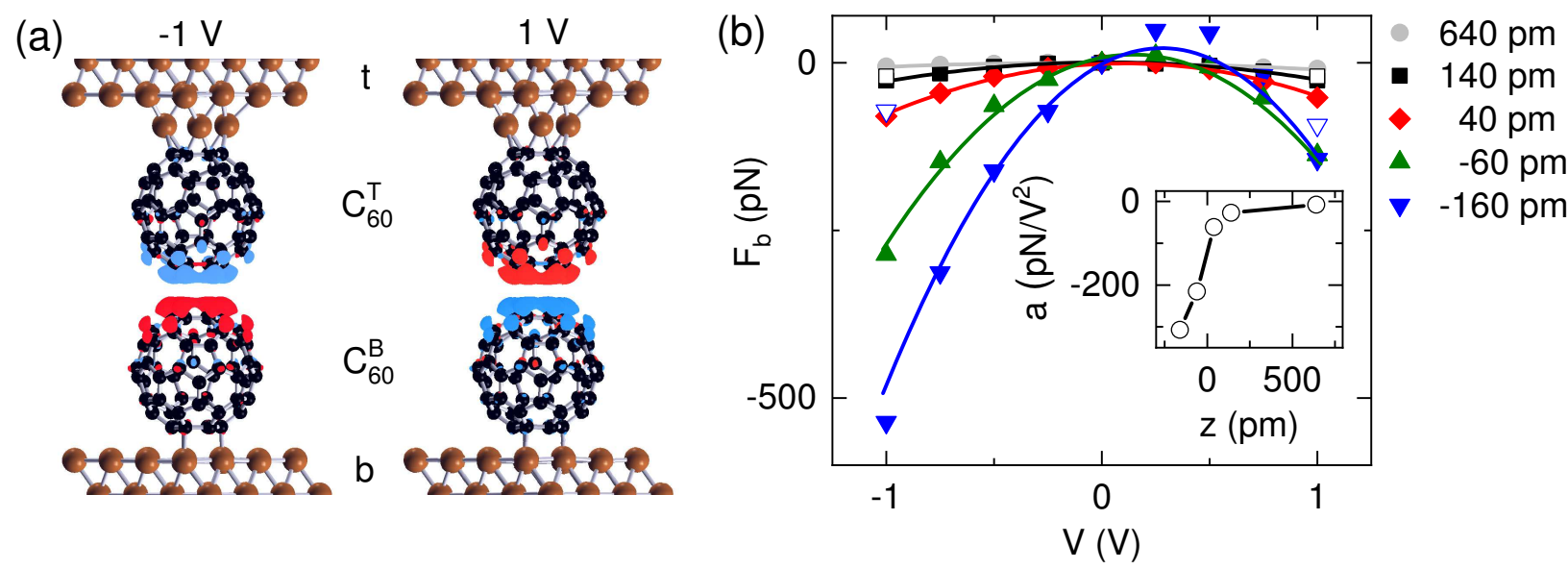

Figure 1: Junction geometry and calculated current-induced bond forces. (a) $\mathrm{Cu}(111)$ electrodes (top: $t$; bottom: $b)$ are modeled by a $(4 \times 4)$ unit cell with periodic boundaries in each electrode plane. Top $\mathrm{C}_{60}\left(\mathrm{C}_{60}^{T}\right)$ is attached via a $\mathrm{C}$ hexagon to $3 \mathrm{Cu}$ atoms arranged in a triangle at nearest-neighbor sites of $\mathrm{Cu}(111)$, while bottom $\mathrm{C}_{60}\left(\mathrm{C}_{60}^{B}\right)$ resides with a $\mathrm{C}$ hexagon on $\mathrm{Cu}(111)$. The calculated accumulated charge density at bias voltage $-1 \mathrm{~V}$ (left), $1 \mathrm{~V}$ (right) is indicated at each $\mathrm{C}_{60}$. (b) Calculated bond force $F_{\mathrm{b}}$ as a function of the bias voltage $V$ for the indicated unrelaxed $\mathrm{C}_{60}-\mathrm{C}_{60}$ distances $z$. Open squares (triangles) depict the electrostatic attraction due to $\mathrm{C}_{60}$ charges (a) at a mutual separation of $140 \mathrm{pm}$ $(-160 \mathrm{pm})$. Inset: Curvature of parabolic fit to $F_{\mathrm{b}}$ as a function of $z$.

and $b$ electrodes with $\mathrm{C}$ hexagons facing each other and are referred to as $\mathrm{C}_{60}^{T}$ and $\mathrm{C}_{60}^{B}$, respectively (the Supporting Information, Figure $\mathrm{S} 1$, show results obtained for $\mathrm{C}_{60}^{T}$ exposing a $\mathrm{C}$ pentagon to the $\mathrm{C}$ hexagon of $\mathrm{C}_{60}^{B}$ ). The chemical potentials $\mu_{t}$ and $\mu_{b}$ of, respectively, $t$ and $b$ electrodes (Figure 1a) differ in order to enable an electric current across the molecular junction. Due to different $\mu_{t}$ and $\mu_{b}$ the $\mathrm{C}_{60}^{T}$ and $\mathrm{C}_{60}^{B}$ molecules exhibit different charges (shaded areas in Figure 1a) that are accumulated at the $\mathrm{C}$ hexagons oriented towards the vacuum. These charges will be used below to estimate the electrostatic attraction between the molecules.

Using the combined DFT-NEGF formalism ${ }^{27,30}$ (Supporting Information) interatomic forces in the $\mathrm{C}_{60}-\mathrm{C}_{60}$ junction were determined from the device Hamiltonian $\mathbf{H}$ and the nonequilibrium electron density defined by the density matrix $\mathbf{D}=\int \mathbf{A}^{t} \cdot n_{\mathrm{F}}\left(E-\mu_{t}\right)-\mathbf{A}^{b}$. $n_{\mathrm{F}}\left(E-\mu_{b}\right) \mathrm{d} E\left(\mathbf{A}^{t, b}:\right.$ spectral functions of $t$ and $b$ electrodes, $n_{\mathrm{F}}$ : Fermi-Dirac function $)$ 
according to ${ }^{27}$

$$
\mathbf{F}_{n}=-\operatorname{Tr}\left(\frac{\partial \mathbf{H}}{\partial \mathbf{r}_{n}} \cdot \mathbf{D}\right)
$$

with $n$ indicating the atom at position $\mathbf{r}_{n}$. Bond forces are defined as the difference of the total forces on $\mathrm{C}_{60}^{T}$ and $\mathrm{C}_{60}^{B}$ projected onto the bond unit vector e connecting the molecular centers of mass, i. e.,

$$
F_{\mathrm{b}}=\left(\sum_{n \in T} \mathbf{F}_{n}-\sum_{n \in B} \mathbf{F}_{n}\right) \cdot \mathbf{e} .
$$

Figure $1 \mathrm{~b}$ shows the bias voltage dependence of $F_{\mathrm{b}}$ for different $\mathrm{C}_{60}-\mathrm{C}_{60}$ distances. Calculated data appear as symbols while solid lines represent fits of a second-order polynomial, $a V^{2}+b V+c$, to the data and serve as a guide to the eye. Distance $z=0 \mathrm{pm}$ is defined at the point of maximum attractive total force in both calculations and experiments (vide infra). This point signals the $\mathrm{C}_{60}-\mathrm{C}_{60}$ chemical bond formation and is referred to as $z_{\mathrm{c}}$ in the following. Distances $z>z_{\mathrm{c}}\left(z<z_{\mathrm{c}}\right)$ correspond to tunneling (contact) ranges.

According to Figure $1 \mathrm{~b}, F_{\mathrm{b}}$ is attractive for essentially all investigated $\mathrm{C}_{60}-\mathrm{C}_{60}$ distances and covered bias voltages. It exhibits a parabolalike evolution with the bias voltage. Figure 1b further reveals that a decreasing mutual molecule distance leads to various effects. First, in the tunneling distance range, $z>140 \mathrm{pm}, F_{\mathrm{b}}$ is comparable to the electrostatic force, $F_{\text {el }}$ (open symbols in Figure $1 \mathrm{~b}$ ), which results from the accumulated charge at $\mathrm{C}_{60}^{T}$ and $\mathrm{C}_{60}^{B}$ (shaded areas in Figure 1a). The upper estimate of $\mathrm{F}_{\mathrm{el}}$ shown in Figure $1 \mathrm{~b}$ was obtained using a plate capacitor model in which the calculated charge densities were attached to discs defined by the planes of the outermost $\mathrm{C}$ hexagons of the two $\mathrm{C}_{60}$. Importantly, entering further into the range of chemical-bond distances, $z<140 \mathrm{pm}, F_{\mathrm{b}}$ outweighs $F_{\mathrm{el}}$ appreciably for finite $V$. Second, with decreasing $\mathrm{C}_{60}-\mathrm{C}_{60}$ separation the parabola apex shifts toward positive $V$ giving rise to an asymmetric behavior of $F_{\mathrm{b}}$. This asymmetry is due to the asymmetric junction setup, i.e., to $\mathrm{C}_{60}^{T}$ adsorbed on a triangular $\mathrm{Cu}$ cluster and $\mathrm{C}_{60}^{B}$ adsorbed on planar $\mathrm{Cu}(111)$. Third, the parabolic curvature, $a$, decreases with decreasing $\mathrm{C}_{60}-\mathrm{C}_{60}$ distance (inset to Figure 1b). A particularly rapid decrease is observed upon entering into distance 
ranges in the vicinity of chemical-bond formation, $z<140 \mathrm{pm}$.

After describing the properties of the calculated bond force $F_{\mathrm{b}}$, its nature will now be elucidated in terms of electron transport across a molecular junction via antibonding and bonding molecular orbitals. ${ }^{32,33}$ The electric current induces a nonequilibrium population of these orbitals and, thus, alters the bond character and strength. The antibonding or bonding character of the bond-forming states is identified by the crystal orbital overlap population (COOP). ${ }^{34}$ It is calculated as the energy-resolved charge density in the intermolecular bond,

$$
\operatorname{COOP}(E)=2 \sum_{i \in T, j \in B} S_{i j} A_{i j}(E)
$$

( $S$ : orbital overlap, $A$ : spectral function in atomic orbital basis), where $i$ and $j$ indicate all $\mathrm{C}_{60}^{T}$ and $\mathrm{C}_{60}^{B}$ orbitals, respectively. The spectral functions may furthermore be partitioned to consider contributions of $t$ and $b$ electrodes $(\alpha=t, b)$ to the origin of scattering states, resulting in decomposed $\operatorname{COOP}^{\alpha}(E)=2 \sum_{i \in T, j \in B} S_{i j} A_{i j}^{\alpha}(E)$. The COOP sign determines whether the states at energy $E$ contributing to the bond have bonding (positive) or antibonding (negative) character. The integration of the $\operatorname{COOP}^{\alpha}$ weighted by $n_{\mathrm{F}}$,

$$
O_{i j}^{\alpha}=S_{i j} \int A_{i j}^{\alpha}(E) n_{\mathrm{F}}\left(E-\mu_{\alpha}\right) \mathrm{d} E
$$

yields the overlap population or bond charge, $\mathrm{OP}=\sum_{\alpha=t, b} \sum_{i \in T, j \in B} O_{i j}^{\alpha}$, which provides a measure of the $\mathrm{C}_{60}-\mathrm{C}_{60}$ bond charge and strength. ${ }^{35}$ The $V$-dependent change of OP with respect to $V=0 \mathrm{~V}$ reads

$$
\Delta \mathrm{OP}=\mathrm{OP}(V)-\mathrm{OP}(0)
$$

Figures 2a,b illustrate the concept of COOP for a $\mathrm{C}_{60}-\mathrm{C}_{60}$ junction at $\left(\mu_{t}-\mu_{b}\right) / \mathrm{e}=-1 \mathrm{~V}$ (e: elementary charge). For positive bias voltage $t$ and $b$ electrodes interchange roles. Figure 2a shows the relevant $\mathrm{C}_{60}$ orbitals involved in the overlap, while Figure $2 \mathrm{~b}$ discloses their bonding or antibonding character, indicated by the shaded areas for, respectively, $E>0 \mathrm{eV}$ 


\section{(a)}

(b)

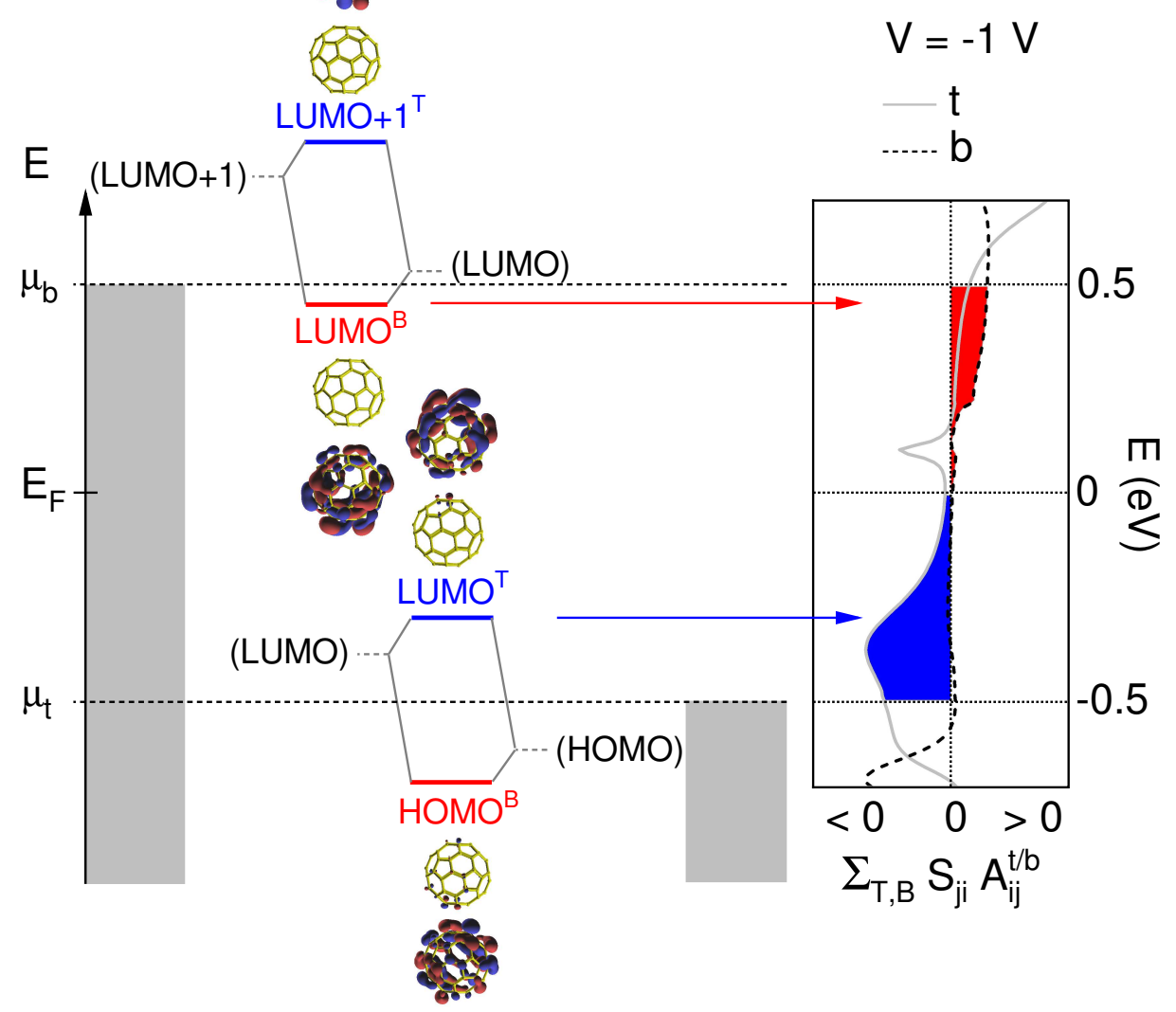

(c)

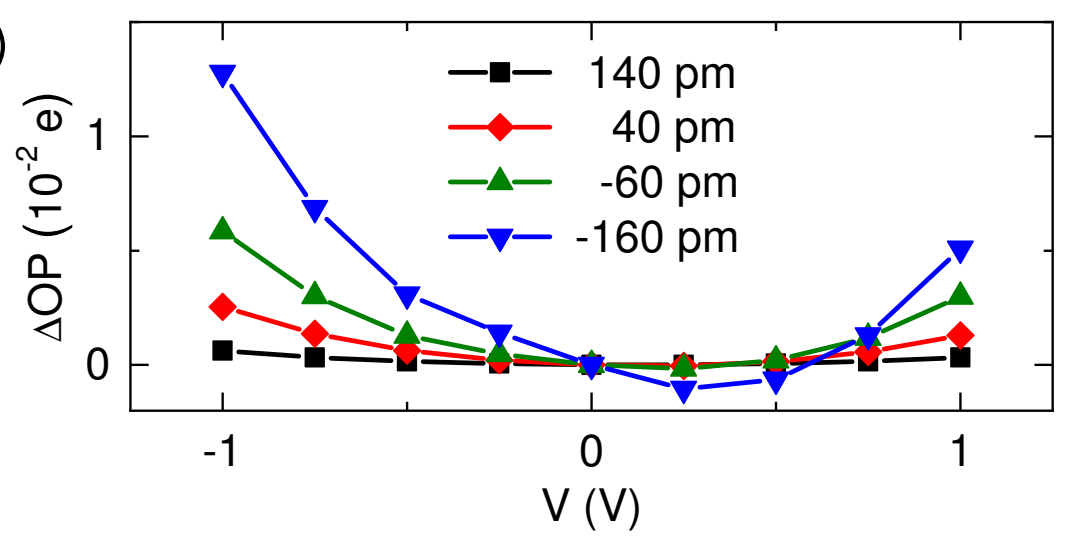

Figure 2: Concept of the crystal orbital overlap population (COOP). (a) Energy levels of the coupled $\mathrm{C}_{60}-\mathrm{C}_{60}$ system $\left(\mathrm{HOMO}^{B}, \mathrm{LUMO}^{T}, \mathrm{LUMO}^{B}, \mathrm{LUMO}+1^{T}\right)$ and the schematic relation to the uncoupled counterparts (HOMO, LUMO, LUMO +1 in parentheses) in a range spanned by the chemical potentials $\mu_{t}$ and $\mu_{b}$ of $t$ and $b$ electrodes for $V=\left(\mu_{t}-\mu_{b}\right) / \mathrm{e}=-1 \mathrm{~V}$. $E_{\mathrm{F}}$ is the equilibrium Fermi energy. Antibonding orbital of $\mathrm{C}_{60}^{T}, \mathrm{LUMO}^{T}$, and bonding orbital of $\mathrm{C}_{60}^{B}, \mathrm{LUMO}^{B}$, fall into the voltage window. (b) Calculated electrode-decomposed COOP (eq 3) of $\mathrm{LUMO}^{T}$ and $\mathrm{LUMO}^{B}$ at $z=-60 \mathrm{pm}$ revealing the bonding (antibonding) character indicated by red (blue) color. $E=0 \mathrm{eV}$ corresponds to $E_{\mathrm{F}}$. The lowering of $\mu_{t}$ depletes the antibonding $\mathrm{LUMO}^{T}$ (blue area), while the increase of $\mu_{b}$ populates the bonding $\mathrm{LUMO}^{B}$ (red area), both resulting in an increase of $\Delta \mathrm{OP}$. (c) $\Delta \mathrm{OP}$ (eq 5) as a function of $V$ for indicated $z$. 
and $E<0 \mathrm{eV}$. Molecular orbitals projected onto the coupled $\mathrm{C}_{60}$ molecules ${ }^{36}$ with energies in the interval $\mu_{t} \leq E_{\mathrm{F}} \leq \mu_{b}\left(E_{\mathrm{F}}\right.$ : equilibrium Fermi energy) are labeled $\mathrm{LUMO}^{T}$ and $\mathrm{LUMO}^{B}$ because of their resemblance with the lowest unoccupied molecular orbital (LUMO) of isolated $\mathrm{C}_{60}$. The resulting electrode-resolved $\operatorname{COOP}^{\alpha}(E)$ is plotted for $t$ and $b$ electrodes in Figure $2 \mathrm{~b}$ as solid and dashed lines, respectively. The highest occupied molecular orbital (HOMO) as well as the LUMO+1 do not participate in the COOP for the considered energies. The scenario depicted in Figures 2a,b is conceptionally close to the simplified model presented previously. ${ }^{32}$

Figure $2 \mathrm{c}$ shows $\Delta \mathrm{OP}$ (eq 5 ) as a function of $V$. For small $\mathrm{C}_{60}-\mathrm{C}_{60}$ distances, $\Delta \mathrm{OP}$ mirrors the nearly quadratic behavior and characteristic asymmetry in the bias voltage polarity as observed from $F_{\mathrm{b}}$ (Figure $1 \mathrm{~b}$ ). At large $\mathrm{C}_{60}-\mathrm{C}_{60}$ distances, however, $\Delta \mathrm{OP}$ is no longer related to $F_{\mathrm{b}}$ reflecting the dominant influence of $F_{\mathrm{el}}$ in that distance range.

Given the akin $V$ dependence of $\Delta \mathrm{OP}$ (Figure $2 \mathrm{c}$ ) and $F_{\mathrm{b}}$ (Figure $1 \mathrm{~b}$ ) at the different $\mathrm{C}_{60}-\mathrm{C}_{60}$ distances, a close correlation between $\Delta \mathrm{OP}$ and $F_{\mathrm{b}}$ is established on the basis of which we attribute the origin of $F_{\mathrm{b}}$ to the bond charge. The $V$-dependent changes in $F_{\mathrm{b}}$ are due to the difference in the chemical potentials, i.e., to the nonequilibrium electron distribution and, thus, to the current. For, e. $g ., V=-1 \mathrm{~V}$ (Figure $2 \mathrm{~b}$ ), $\mu_{b}>\mu_{t}$ leads to an increased population of the bonding $\mathrm{LUMO}^{B}$ (red area) and a concomitantly decreased population of the antibonding $\mathrm{LUMO}^{T}$ (blue area) with respect to the equilibrium situation at $0 \mathrm{~V}$, which both increase $\Delta \mathrm{OP}$ and give rise to a current-induced $\mathrm{C}_{60}-\mathrm{C}_{60}$ attraction. Moreover, the junction electric field affects the level alignment ${ }^{37,38}$ and effectively increases the electronic coupling and, thus, enhances the discussed effect with increasing $V$.

Combined AFM and STM experiments (Supporting Information) were carried out to test the theoretical results. Using a surface science approach, junctions were assembled in situ to mimic the setup in the model calculations as closely as possible. To this end, $\mathrm{C}_{60}$ was adsorbed on $\mathrm{Cu}(111)$ (Figure 3a). Ordered molecular islands with monolayer (ML) height were obtained after annealing the $\mathrm{C}_{60}$-covered sample. Separate $\mathrm{C}_{60}$ molecules were detached 

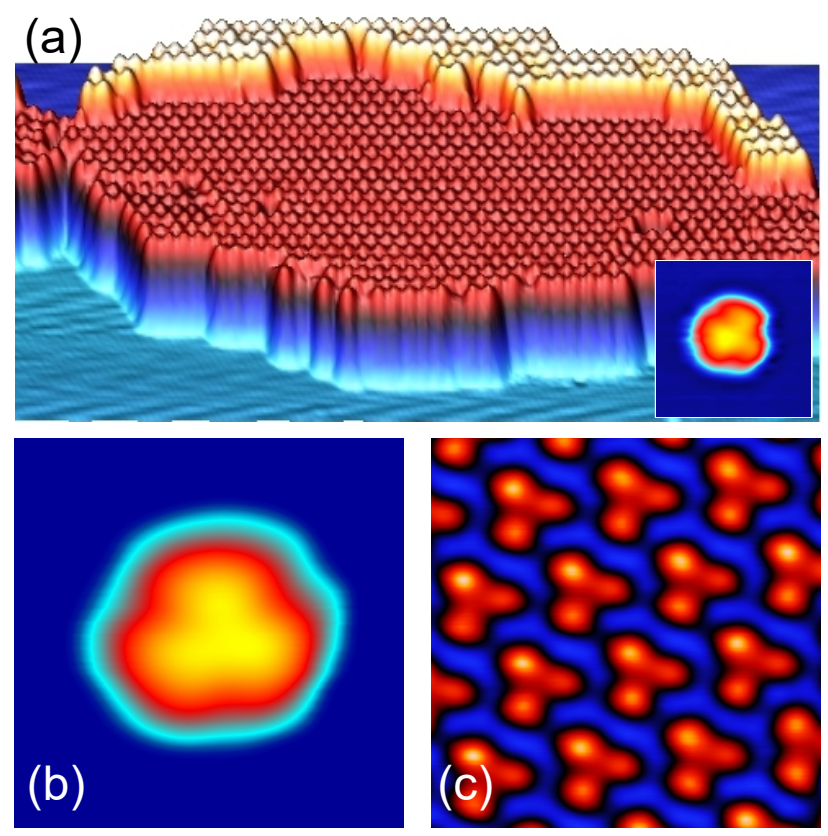

Figure 3: STM topographic data of $\mathrm{C}_{60}$-covered $\mathrm{Cu}(111)$ and of a $\mathrm{C}_{60}$-terminated tip. (a) Constant-current STM image of a $\mathrm{C}_{60}$ island on $\mathrm{Cu}(111)\left(0.7 \mathrm{~V}, 100 \mathrm{pA}, 50 \times 25 \mathrm{~nm}^{2}\right)$. The color scale ranges from $0 \mathrm{pm}$ (blue) to $830 \mathrm{pm}$ (white). Inset: $\mathrm{STM}$ image of a $\mathrm{C}_{60}$-terminated tip using an atomic protrusion on $\mathrm{Cu}(111)\left(-2.1 \mathrm{~V}, 50 \mathrm{pA}, 2.2 \times 2.2 \mathrm{~nm}^{2}\right)$. The color scale ranges from $0 \mathrm{pm}$ (dark blue) to $100 \mathrm{pm}$ (bright yellow). (b) Close-up view of a separated $\mathrm{C}_{60}\left(1.7 \mathrm{~V}, 50 \mathrm{pA}, 4 \times 4 \mathrm{~nm}^{2}\right)$. (c) Close-up view of the inner part of a $\mathrm{C}_{60}$ island $(2 \mathrm{~V}, 50 \mathrm{pA}$, $3 \times 3 \mathrm{~nm}^{2}$ ). For (b), (c) the same color scale as in (a) is used. STM data were processed using WSXM. ${ }^{39}$ 
from the step edges of ML molecular islands and dragged across the substrate terrace with the tip of the microscope. Close-up views of separate $\mathrm{C}_{60}$ molecules (Figure $3 \mathrm{~b}$ ) and $\mathrm{ML}$ islands (Figure 3c) reveal a trefoillike structural motif of adsorbed $\mathrm{C}_{60}$, which indicates a $\mathrm{C}$ hexagon exposed to vacuum and to the surface, in agreement with a previous report. ${ }^{40}$ The STM data of Figure 3 were acquired with a PtIr tip whose apex had presumably been coated with $\mathrm{Cu}$ substrate material due to the applied tip preparation protocol (Supporting Information). For the force measurements to be discussed next, the tip was terminated with a single $\mathrm{C}_{60}$ molecule using a standard procedure. ${ }^{41,42}$ The successful transfer to the tip was verified by imaging the modified tip apex with an adsorbed $\mathrm{Cu}$ atom that had been transferred from the $\mathrm{Cu}$-coated tip to $\mathrm{Cu}(111)^{43-46}$ prior to $\mathrm{C}_{60}$ termination of the tip apex. The inset to Figure 3a shows an STM image revealing that $\mathrm{C}_{60}$ at the tip is likewise oriented with a $\mathrm{C}$ hexagon to the vacuum.

Figure 4a presents the changes in the tuning fork resonance frequency $\Delta f$ during the approach of a $\mathrm{C}_{60}$-terminated tip to a single separated $\mathrm{C}_{60}$, i. e., for decreasing $z$. The same plot contains the vertical force, $F_{z}$, extracted from $\Delta f$ according to previously reported algorithms, ${ }^{47,48}$ which led to virtually identical results. The evolution of the simultaneously recorded current, $I$, is shown in Figure $4 \mathrm{~b}$.

$F_{z}(z)$ (Figure $4 \mathrm{a}$ ) adopts the expected Lennard-Jones-type ${ }^{49,50}$ behavior. At large $z$, $F_{z}(z)$ is dominated by the long-range and slowly varying van der Waals force and electrostatic attraction between the electrodes. The minimum of $F_{z}$ at $z_{\mathrm{c}}^{(F)}$ signals the point of maximum attraction and defines the distance range for chemical-bond formation. For $z<z_{\mathrm{c}}^{(F)}$ the short-range Pauli repulsion progressively strengthens and, thereby, weakens the $\mathrm{C}_{60}-\mathrm{C}_{60}$ attraction. Figure $4 \mathrm{~b}$ shows the current evolution with $z$ around $z=0 \mathrm{pm} . I(z)$ increases exponentially with decreasing $\mathrm{C}_{60}-\mathrm{C}_{60}$ separation, more strongly for $z>z_{\mathrm{c}}^{(I)}$ than for $z<$ $z_{\mathrm{c}}^{(I)}$. The intersection of the extrapolated exponential fits to the data (dashed lines in Figure 4b) approximates the contact point, $z_{\mathrm{c}}^{(I)}{ }^{43,45,51,52}$ Both data sets, $F_{z}(z)$ and $I(z)$, provide a consistent definition of the contact point, i.e., $z_{c} \equiv z_{\mathrm{c}}^{(F)} \approx z_{\mathrm{c}}^{(I)}$. Similar behavior was 

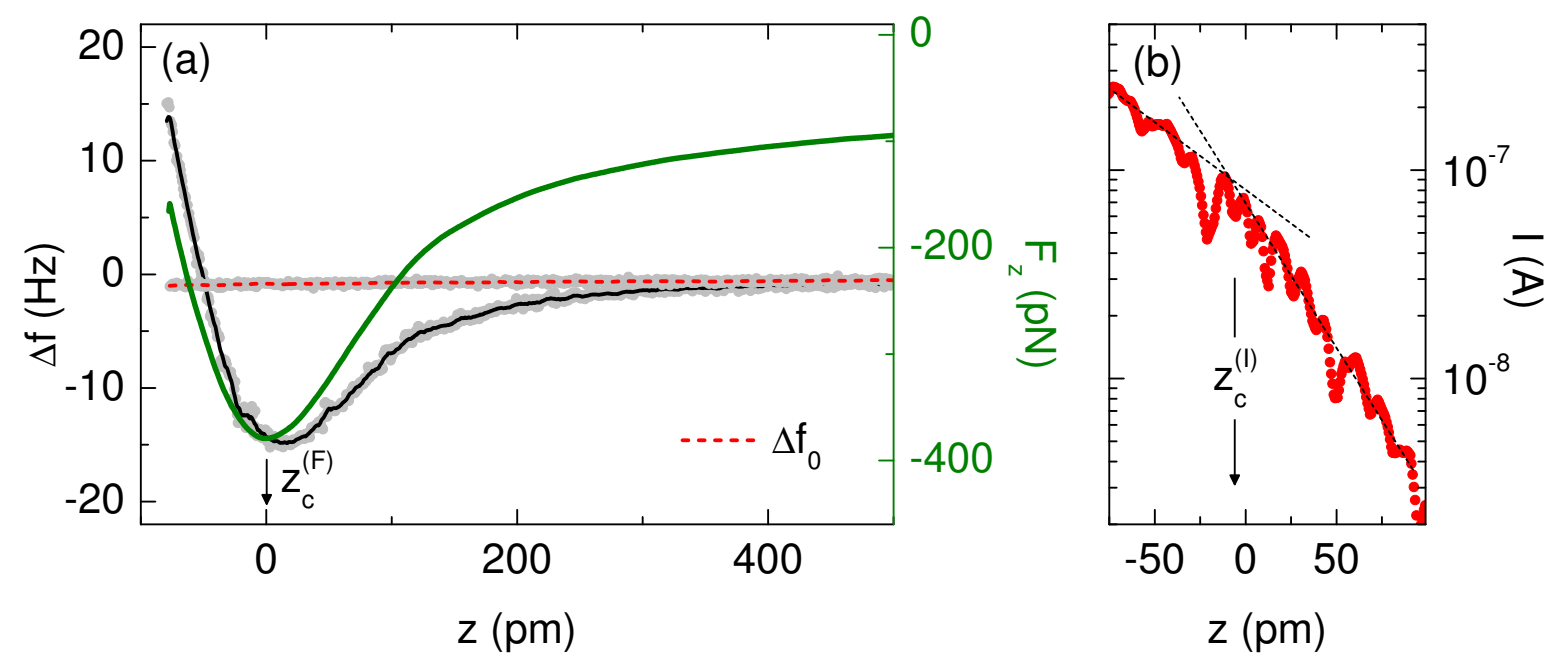

Figure 4: Force and current data obtained from $\mathrm{C}_{60}-\mathrm{C}_{60}$ junctions. (a) Resonance frequency change $\Delta f$ (gray dots and superimposed smoothed data as a black line) and vertical force $F_{z}$ (green line) as a function of $z$ measured at $0.6 \mathrm{~V}$ for a $\mathrm{C}_{60}$-terminated tip and a single $\mathrm{C}_{60}$ on $\mathrm{Cu}(111)$. The minimum of $F_{z}$ at $z_{\mathrm{c}}^{(F)}$ signals $\mathrm{C}_{60}-\mathrm{C}_{60}$ contact formation. $\Delta f_{0}$ data (gray dots and superimposed smoothed data as a dashed line) were obtained with the same $\mathrm{C}_{60^{-}}$ terminated tip on clean $\mathrm{Cu}(111)$. An additional $z$ offset was applied to guarantee the same absolute tip-surface distance as for $\Delta f(z)$ measurements atop adsorbed $\mathrm{C}_{60}$. (b) Current $I$ (dots) across the $\mathrm{C}_{60}-\mathrm{C}_{60}$ junction of (a) acquired simultaneously with $\Delta f$. Dashed lines are exponential fits to $I(z)$ data for $z<z_{\mathrm{c}}^{(I)}$ and $z>z_{\mathrm{c}}^{(I)}$ whose intersection indicate the point of contact at $z_{\mathrm{c}}^{(I)}$. In (a), (b) the feedback loop had been disabled at $1 \mathrm{~V}, 50 \mathrm{pA}$ and the bias voltage was subsequently ramped to $0.6 \mathrm{~V}$. 
observed from $\mathrm{C}_{60}$ molecules embedded in ML molecular islands (not shown).

In order to access the predicted bond forces $F_{\mathrm{b}}$ (Figure $1 \mathrm{~b}$ ) long-range, slowly varying attractive forces between the full tip structure and surface must be removed from the experimentally obtained total force $F_{z}$ (Figure $4 \mathrm{a}$ ). The following approximation has to be made to this end. The measured total force acting on the tip, $F_{z}$, can be decomposed into a short-ranged force between the two $\mathrm{C}_{60}$ molecules, $F_{z}^{*}$, and a residual long-ranged and slowly varying force, $F_{0}$, acting between the tip including the attached $\mathrm{C}_{60}$ and the metal substrate. One may expect $F_{z}^{*}$ and its bias voltage dependence to be negligible at large distance, while at chemical-bond distances only $F_{z}^{*}$ will reflect the charge redistributions occuring at the $\mathrm{C}_{60}$ molecules in the course of the current-induced population of bonding and antibonding orbitals. In contrast, $F_{0}$ takes into account charges well separated from the $\mathrm{C}_{60}-\mathrm{C}_{60}$ junction and is independent of the presence of $\mathrm{C}_{60}$ adsorbed on $\mathrm{Cu}(111)$.

To obtain $F_{z}^{*}$ and test this approximation, we follow a previously suggested and successfully applied procedure ${ }^{25,53,54}$ where the resonance frequency changes were acquired with a $\mathrm{C}_{60}$-terminated tip on an extended pristine $\mathrm{Cu}(111)$ surface region $\left(\approx 200 \times 200 \mathrm{~nm}^{2}\right)$ at the same absolute tip-surface distance as used for the $\Delta f(z)$ measurements atop adsorbed $\mathrm{C}_{60}$ on $\mathrm{Cu}(111)$. These frequency changes are referred to as $\Delta f_{0}$ (dashed line in Figure 4a) and reflect the long-ranged slowly varying force $F_{0}$ (vide supra). The subtraction of the long-range and slowly varying $F_{0}$ from the total force $F_{z}$ is performed by transforming $\Delta f-\Delta f_{0}$ into a force, ${ }^{47,48} F_{z}^{*}$. This approach was used for correcting $\Delta f$ and $F_{z}$ obtained for separated $\mathrm{C}_{60}$ as well as for molecules embedded in an ML island. In Figure $5 F_{z}^{*}$ is plotted as a function of $V$ and different $\mathrm{C}_{60}-\mathrm{C}_{60}$ distances for the single (Figure 5a) and ML (Figure $5 \mathrm{~b}$ ) $\mathrm{C}_{60}$. The vertical offset is due to the distance-dependent short-range force.

In all cases $F_{z}^{*}$ is essentially independent of $V$ for large mutual $\mathrm{C}_{60}$ distances (top), which reflects the appropriate subtraction of long-range and slowly varying forces, as expected. With decreasing $\mathrm{C}_{60}-\mathrm{C}_{60}$ distance $F_{z}^{*}$ exhibits a progressively pronounced parabolalike behavior (middle and bottom). In order to facilitate the comparison with the calculated results 

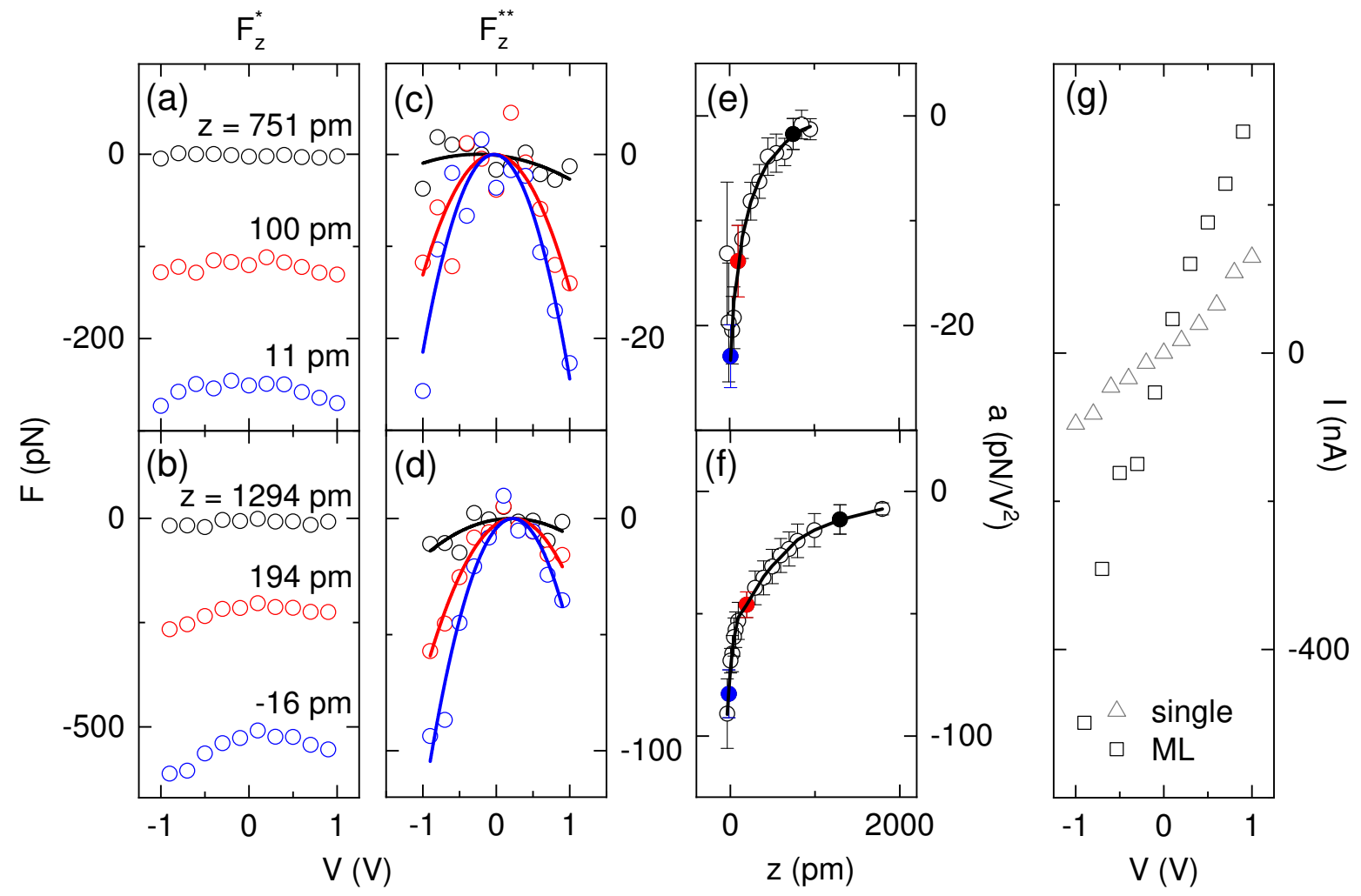

Figure 5: Experimental evidence for current-induced forces. (a), (b) Residual force $F_{z}^{*}$ for (a) single and (b) $\mathrm{ML} \mathrm{C}_{60}$ obtained for the indicated distances $z$ after subtracting long-range forces from $F_{z}$ (see text). (c), (d) Plots of $F_{z}^{* *}(V)=F_{z}^{*}(V)-F_{z}^{*}(0)$ for (c) single and (d) ML $\mathrm{C}_{60}$. Solid lines depict parabolic fits to $F_{z}^{* *}$. (e), (f) Parabola curvatures of (c), (d) for different $z$. Dots correspond to the data presented in (a)-(d). (g) $I-V$ characteristics for single (triangles) and ML (squares) $\mathrm{C}_{60}$ acquired at $z=0 \mathrm{pm}$. 
(Figure $1 \mathrm{~b}) F_{z}^{*}$ is referred to its value at the parabola maximum close to $0 \mathrm{~V}$, resulting in $F_{z}^{* *}(V)=F_{z}^{*}(V)-F_{z}^{*}(V \approx 0 \mathrm{~V})$ plotted for single and $\mathrm{ML} \mathrm{C}_{60}$ in Figure $5 \mathrm{c}$ and Figure $5 \mathrm{~d}$, respectively. The solid lines represent parabolic fits to the data with the curvature plotted as a function of $z$ in Figure 5e,f.

How do the experimental data compare with the theoretical findings? The experimentally extracted $F_{z}^{* *}$ is in qualitative agreement with the calculated $F_{\mathrm{b}}$. Both $F_{z}^{* *}$ and $F_{\mathrm{b}}$ are attractive and exhibit a nearly parabolic evolution with $V$ (Figure 1b, Figure 5c,d). Their curvature is negative, weak in the far tunneling range and increasingly pronounced for smaller $\mathrm{C}_{60}-\mathrm{C}_{60}$ distances. Moreover, the curvature rapidly decreases upon entering into the range of chemical-bond distances in both cases (inset to Figure 1b, Figure 5e,f). Quantitatively, however, there are some deviations between experiment and theory. The calculated properties generally tend to overestimate the experimental results since only the $\mathrm{C}_{60}-\mathrm{C}_{60}$ bond force is accounted for. The additional compliance due to relaxations of tip and surface structures is not considered. In addition, periodic boundary conditions are used in the simulations that inevitably lead to multiple tips in the model setup, which contribute to the overestimation of currents and forces (Supporting Information, Figure S2). The junction conductance in the calculations is approximately two times larger than the experimentally observed conductance, which entails a correspondingly larger bond force in the model than in the experiments. For instance, in the vicinity of chemical-bond distances, $z \approx z_{\mathrm{c}}, F_{z}^{* *}$ varies between $0 \mathrm{pN}$ and $\approx-25 \mathrm{pN}$ for single $\mathrm{C}_{60}$ (Figure $5 \mathrm{c}$ ) and between $0 \mathrm{pN}$ and $\approx-100 \mathrm{pN}$ for ML $\mathrm{C}_{60}$ in the voltage interval $-1 \mathrm{~V} \leq V \leq 1 \mathrm{~V}$. The corresponding variation of $F_{\mathrm{b}}$ covers the range from $0 \mathrm{pN}$ to $\approx-100 \mathrm{pN}$ and thus is a factor $\approx 4$ larger than observed for single $\mathrm{C}_{60}$, while it matches the range of $F_{z}^{* *}$ for $\mathrm{ML}_{60}$ very well. In addition, the evolution of the $F_{\mathrm{b}}(V)$ curvature is more pronounced in the calculations than for the measured $F_{z}^{* *}(V)$. The abrupt decrease of the calculated curvature upon entering chemical-bond distances is smoothed in the experiments due to, supposedly, a less stiff junction in which relaxations of tip- $\mathrm{C}_{60}$ and $\mathrm{C}_{60}$-surface geometries are involved. Because of the overall qualitative and 
in part quantitative accordance between $F_{z}^{* *}$ and $F_{\mathrm{b}}$ ample evidence is provided by the experiment for the presence of the calculated current-induced forces.

The importance of the current is likewise evidenced by the comparison of $I-V$ characteristics (Figure $5 \mathrm{~g}$ ) with $F_{z}^{* *}$. In the relevant range of bias voltages, $-1 \mathrm{~V} \leq V \leq 1 \mathrm{~V}$, the current across single $\mathrm{C}_{60}$ (triangles) is a factor $\approx 4$ lower than across $\mathrm{C}_{60}$ embedded in ML islands (squares), which matches well the ratio of $F_{z}^{* *}$ data obtained for these junctions (Figure $5 \mathrm{c}, \mathrm{d}$ ). The difference in the $I-V$ characteristics is most likely due to the stronger $\mathrm{C}_{60}-\mathrm{Cu}(111)$ hybridization in the molecular island where the substrate surface exhibits a $\mathrm{C}_{60}$-induced reconstruction. ${ }^{55}$ As a consequence, the $\mathrm{C}_{60}$ LUMO is located at the Fermi energy $^{55}$ (Supporting Information, Figure S3), which entails a higher junction conductance than observed for single $\mathrm{C}_{60}$. Furthermore, $I-V$ data for $\mathrm{ML}_{60}$ exhibit a more pronounced asymmetry with the polarity of the bias voltage. This behavior is likewise present for $F_{z}^{* *}$. In the calculations the $F_{\mathrm{b}}$ asymmetry with the bias voltage polarity was assigned to the asymmetric coupling of $\mathrm{C}_{60}$ to the tip and to the substrate. However, the degree of asymmetry in the molecule-electrode coupling may considerably be reduced in the experiments. The metal tip may be less sharp than assumed in the calculations. In this case it would therefore represent a rather flat adsorption region for $\mathrm{C}_{60}$, which is similar to the adsorption situation for $\mathrm{C}_{60}$ on the counter electrode, $\mathrm{Cu}(111)$.

Before concluding it is noteworthy that deviations from $\mathrm{C}_{60}$ hexagon orientations at the tip apex did not change the characteristics of the bond force significantly. In the Supporting Information (Figure S4) $F_{z}^{*}$ and $F_{z}^{* *}$ are presented for a tip terminated by a $\mathrm{C}_{60}$ molecule exposing a $\mathrm{C}$ pentagon to the surface. The weak dependence of $F_{z}^{*}, F_{z}^{* *}$ on the $\mathrm{C}_{60}$ orientation is in agreement with simulations (Supporting Information, Figure S1). The akin behavior may tentatively be attributed to the spatial distribution of the $\mathrm{C}_{60}$ LUMO, which is most important for contact formation (Figure 2a). It exhibits a toruslike shape centered at the C pentagon. ${ }^{45,51}$ The $\mathrm{C}$ hexagon orientation of $\mathrm{C}_{60}$ still shows good parts of the LUMO of the adjacent three $\mathrm{C}$ pentagons (Figure 3b,c). Therefore, the LUMO overlap may be 
comparable in case of the different $\mathrm{C}_{60}$ orientations considered here and result in the similar behavior of the bond force. Another rationale may be a favored contact geometry; that is, in the Pauli repulsion distance range the reaction partners adopt a preferred orientation for bond formation that is independent of the initial orientation prior to contact. Forthcoming experiments will involve $\mathrm{C}_{60}$ on $\mathrm{Cu}(100)$ where several orientations of adsorbed $\mathrm{C}_{60}$ are available. $^{51}$

In conclusion, theory and experiment unambiguously unveil current-induced bond forces in a molecular contact and contribute to the understanding of the relation between force and current in nanometer-scaled junctions. Owing to the assembly of the junction with precise control of the electrode composition and structure, model calculations and force spectroscopy experiments are based on closely matching contact geometries and, thus, enable a meaningful comparison of calculated and measured data. The current-induced bond forces in a biased conductive single-molecule junction result from the nonequilibrium population of bonding and antibonding molecular orbitals. These forces exhibit a nearly parabolic bias voltage behavior and are dominant for molecule-molecule separations in the chemical-bond distance range. Their magnitude depends on the orbital overlap in the energy range defined by the electrode chemical potentials. The reported theoretical and experimental findings are relevant to miniaturized circuits in molecular electronics and offer a fresh view on the formation of chemical bonds at the single-molecule level in the presence of a current.

\section{Acknowledgement}

Funding by the Deutsche Forschungsgemeinschaft through Grant No. KR 2912/12-1, Villum Fonden (Grant No. 00013340) and the Danish Research Foundation (Project DNRF103) is acknowledged. 


\section{Supporting Information Available}

The Supporting Information is available free of charge on the ACS Publications website at

DOI: [hyperlink DOI]

Details on DFT modeling, NEGF calculation, data for different $\mathrm{C}_{60}$ orientations, sample and tip preparation, spectroscopy of $\mathrm{d} / \mathrm{d} V$

\section{References}

(1) Kaiser, U.; Schwarz, A.; Wiesendanger, R. Magnetic exchange force microscopy with atomic resolution. Nature 2007, 446, 522-525.

(2) Grenz, J.; Köhler, A.; Schwarz, A.; Wiesendanger, R. Probing the Nano-Skyrmion Lattice on Fe/Ir(111) with Magnetic Exchange Force Microscopy. Phys. Rev. Lett. 2017, 119,047205 .

(3) Ternes, M.; Lutz, C. P.; Hirjibehedin, C. F.; Giessibl, F. J.; Heinrich, A. J. The Force Needed to Move an Atom on a Surface. Science 2008, 319, 1066-1069.

(4) Gross, L.; Mohn, F.; Moll, N.; Liljeroth, P.; Meyer, G. The Chemical Structure of a Molecule Resolved by Atomic Force Microscopy. Science 2009, 325, 1110-1114.

(5) de Oteyza, D. G.; Gorman, P.; Chen, Y.-C.; Wickenburg, S.; Riss, A.; Mowbray, D. J.; Etkin, G.; Pedramrazi, Z.; Tsai, H.-Z.; Rubio, A.; Crommie, M. F.; Fischer, F. R. Direct Imaging of Covalent Bond Structure in Single-Molecule Chemical Reactions. Science 2013, 340, 1434-1437.

(6) Schneiderbauer, M.; Emmrich, M.; Weymouth, A. J.; Giessibl, F. J. CO Tip Functionalization Inverts Atomic Force Microscopy Contrast via Short-Range Electrostatic Forces. Phys. Rev. Lett. 2014, 112, 166102. 
(7) Corso, M.; Ondráček, M.; Lotze, C.; Hapala, P.; Franke, K. J.; Jelínek, P.; Pascual, J. I. Charge Redistribution and Transport in Molecular Contacts. Phys. Rev. Lett. 2015, $115,136101$.

(8) Weymouth, A. J.; Wutscher, T.; Welker, J.; Hofmann, T.; Giessibl, F. J. Phantom Force Induced by Tunneling Current: A Characterization on Si(111). Phys. Rev. Lett. 2011, 106, 226801.

(9) Landauer, R.; Woo, J. W. F. Driving force in electromigration. Phys. Rev. B 1974, 10, $1266-1271$.

(10) Das, A. K.; Peierls, R. The force of electromigration. J. Phys. C: Solid State Phys. 1975, 8, 3348-3352.

(11) Sham, L. J. Microscopic theory of the driving force in electromigration. Phys. Rev. B 1975, 12, 3142-3149.

(12) Sorbello, R. S. In Solid State Physics; Ehrenreich, H., Spaepen, F., Eds.; Academic Press, 1997; Vol. 51; and references therein.

(13) Yang, Z.; Di Ventra, M. Nonlinear current-induced forces in Si atomic wires. Phys. Rev. B 2003, 67, 161311.

(14) Di Ventra, M.; Pantelides, S. T.; Lang, N. D. Current-Induced Forces in Molecular Wires. Phys. Rev. Lett. 2002, 88, 046801.

(15) Verdozzi, C.; Stefanucci, G.; Almbladh, C.-O. Classical Nuclear Motion in Quantum Transport. Phys. Rev. Lett. 2006, 97, 046603.

(16) Seideman, T. Current-driven dynamics in molecular-scale devices. J. Phys.: Condens. Matter 2003, 15, R521-R549.

(17) Hettler, M. H.; Wenzel, W.; Wegewijs, M. R.; Schoeller, H. Current Collapse in Tunneling Transport through Benzene. Phys. Rev. Lett. 2003, 90, 076805. 
(18) Huang, Z.; Chen, F.; D’agosta, R.; Bennett, P. A.; Di Ventra, M.; Tao, N. Local ionic and electron heating in single-molecule junctions. Nat. Nanotechnol. 2007, 2, 698 703.

(19) Dundas, D.; McEniry, E. J.; Todorov, T. N. Current-driven atomic waterwheels. Nat. Nanotechnol. 2009, 4, $99-102$.

(20) Tierney, H. L.; Murphy, C. J.; Jewell, A. D.; Baber, A. E.; Iski, E. V.; Khodaverdian, H. Y.; McGuire, A. F.; Klebanov, N.; Sykes, E. C. H. Experimental demonstration of a single-molecule electric motor. Nat. Nanotechnol. 2011, 6, 625 - 629.

(21) Lü, J.-T.; Brandbyge, M.; Hedegård, P.; Todorov, T. N.; Dundas, D. Current-induced atomic dynamics, instabilities, and Raman signals: Quasiclassical Langevin equation approach. Phys. Rev. B 2012, 85, 245444.

(22) Li, Y.; Doak, P.; Kronik, L.; Neaton, J. B.; Natelson, D. Voltage tuning of vibrational mode energies in single-molecule junctions. Proc. Natl. Acad. Sci. 2014, 111, 12821287.

(23) Hsu, B. C.; Amanatidis, I.; Liu, W.-L.; Tseng, A.; Chen, Y.-C. Effects of CurrentInduced Forces on Pt-Benzene-Pt Single-Molecule Junctions. J. Phys. Chem. C 2014, $118,2245-2252$.

(24) Bai, M.; Cucinotta, C. S.; Jiang, Z.; Wang, H.; Wang, Y.; Rungger, I.; Sanvito, S.; Hou, S. Current-induced phonon renormalization in molecular junctions. Phys. Rev. B 2016, 94, 035411.

(25) Ternes, M.; González, C.; Lutz, C. P.; Hapala, P.; Giessibl, F. J.; Jelínek, P.; Heinrich, A. J. Interplay of Conductance, Force, and Structural Change in Metallic Point Contacts. Phys. Rev. Lett. 2011, 106, 016802. 
(26) Perdew, J. P.; Burke, K.; Ernzerhof, M. Generalized Gradient Approximation Made Simple. Phys. Rev. Lett. 1996, 77, 3865-3868.

(27) Brandbyge, M.; Mozos, J.-L.; Ordejón, P.; Taylor, J.; Stokbro, K. Density-functional method for nonequilibrium electron transport. Phys. Rev. B 2002, 65, 165401.

(28) Grimme, S.; Antony, J.; Ehrlich, S.; Krieg, H. A consistent and accurate ab initio parametrization of density functional dispersion correction (DFT-D) for the 94 elements H-Pu. J. Chem. Phys. 2010, 132, 154104.

(29) Hu, Z.-X.; Lan, H.; Ji, W. Role of the dispersion force in modeling the interfacial properties of molecule-metal interfaces: adsorption of thiophene on copper surfaces. Sci. Rep. 2014, 4, 5036.

(30) Papior, N.; Lorente, N.; Frederiksen, T.; García, A.; Brandbyge, M. Improvements on non-equilibrium and transport Green function techniques: The next-generation TRANSIESTA. Comput. Phys. Commun. 2017, 212, 8-24.

(31) Papior, N. sisl: v0.9.4. 2018; https://doi.org/10.5281/zenodo.597181.

(32) Brandbyge, M.; Stokbro, K.; Taylor, J.; Mozos, J.-L.; Ordejón, P. Origin of currentinduced forces in an atomic gold wire: A first-principles study. Phys. Rev. B 2003, 67, 193104.

(33) Leitherer, S.; Papior, N.; Brandbyge, M. Current-induced atomic forces in gated graphene nanoconstrictions. Phys. Rev. B 2019, 100, 035415.

(34) Hoffmann, R. A chemical and theoretical way to look at bonding on surfaces. Rev. Mod. Phys. 1988, 60, 601-628.

(35) Mulliken, R. S. Electronic Population Analysis on LCAO-MO Molecular Wave Functions. I. J. Chem. Phys. 1955, 23, 1833-1840. 
(36) Stokbro, K.; Taylor, J.; Brandbyge, M.; Mozos, J.-L.; Ordejón, P. Theoretical study of the nonlinear conductance of Di-thiol benzene coupled to $\mathrm{Au}(111)$ surfaces via thiol and thiolate bonds. Comput. Mater. Sci. 2003, 27, 151 - 160, E-MRS Symposium Spring Meeting - Symposium A: Atomic Scale Materials Design.

(37) Limot, L.; Maroutian, T.; Johansson, P.; Berndt, R. Surface-State Stark Shift in a Scanning Tunneling Microscope. Phys. Rev. Lett. 2003, 91, 196801.

(38) Kröger, J.; Limot, L.; Jensen, H.; Berndt, R.; Johansson, P. Stark effect in Au(111) and $\mathrm{Cu}(111)$ surface states. Phys. Rev. B 2004, 70, 033401.

(39) Horcas, I.; Fernández, R.; Gómez-Rodríguez, J. M.; Colchero, J.; Gómez-Herrero, J.; Baro, A. M. WSXM: A software for scanning probe microscopy and a tool for nanotechnology. Rev. Sci. Instrum. 2007, 78, 013705.

(40) Larsson, J. A.; Elliott, S. D.; Greer, J. C.; Repp, J.; Meyer, G.; Allenspach, R. Orientation of individual $\mathrm{C}_{60}$ molecules adsorbed on $\mathrm{Cu}(111)$ : Low-temperature scanning tunneling microscopy and density functional calculations. Phys. Rev. B 2008, 77, 115434.

(41) Kelly, K. F.; Sarkar, D.; Prato, S.; Resh, J. S.; Hale, G. D.; Halas, N. J. Direct observation of fullerene-adsorbed tips by scanning tunneling microscopy. J. Vac. Sci. Technol. B 1996, 14, 593-596.

(42) Schull, G.; Frederiksen, T.; Arnau, A.; Sanchez-Portal, D.; Berndt, R. Atomic-scale engineering of electrodes for single-molecule contacts. Nat. Nanotechnol. 2011, 6, 2327.

(43) Limot, L.; Kröger, J.; Berndt, R.; Garcia-Lekue, A.; Hofer, W. A. Atom Transfer and Single-Adatom Contacts. Phys. Rev. Lett. 2005, 94, 126102.

(44) Kröger, J.; Jensen, H.; Berndt, R. Conductance of tip-surface and tip-atom junctions on $\mathrm{Au}(111)$ explored by a scanning tunnelling microscope. New J. Phys. 2007, 9, 153. 
(45) Kröger, J.; Néel, N.; Limot, L. Contact to single atoms and molecules with the tip of a scanning tunnelling microscope. J. Phys.: Condens. Matter 2008, 20, 223001.

(46) Kröger, J.; Néel, N.; Sperl, A.; Wang, Y. F.; Berndt, R. Single-atom contacts with a scanning tunnelling microscope. New J. Phys. 2009, 11, 125006.

(47) Giessibl, F. J. A direct method to calculate tip-sample forces from frequency shifts in frequency-modulation atomic force microscopy. Appl. Phys. Lett. 2001, 78, 123-125.

(48) Sader, J. E.; Jarvis, S. P. Accurate formulas for interaction force and energy in frequency modulation force spectroscopy. Appl. Phys. Lett. 2004, 84, 1801-1803.

(49) Pawlak, R.; Kawai, S.; Fremy, S.; Glatzel, T.; Meyer, E. Atomic-Scale Mechanical Properties of Orientated C60 Molecules Revealed by Noncontact Atomic Force Microscopy. ACS Nano 2011, 5, 6349-6354, PMID: 21736339.

(50) Hauptmann, N.; Mohn, F.; Gross, L.; Meyer, G.; Frederiksen, T.; Berndt, R. Force and conductance during contact formation to a C 60 molecule. New J. Phys. 2012, 14 , 073032 .

(51) Néel, N.; Kröger, J.; Limot, L.; Frederiksen, T.; Brandbyge, M.; Berndt, R. Controlled Contact to a $\mathrm{C}_{60}$ Molecule. Phys. Rev. Lett. 2007, 98, 065502.

(52) Berndt, R.; Kröger, J.; Néel, N.; Schull, G. Controlled single atom and single molecule contacts. Phys. Chem. Chem. Phys. 2010, 12, 1022-1032.

(53) Ladenthin, J. N.; Frederiksen, T.; Persson, M.; Sharp, J. C.; Gawinkowski, S.; Waluk, J.; Kumagai, T. Force-induced tautomerization in a single molecule. Nat. Chem. 2016, 8, $935-940$.

(54) Liebig, A.; Giessibl, F. J. In-situ characterization of O-terminated Cu tips for highresolution atomic force microscopy. Appl. Phys. Lett. 2019, 114, 143103. 
(55) Pai, W. W.; Jeng, H. T.; Cheng, C.-M.; Lin, C.-H.; Xiao, X.; Zhao, A.; Zhang, X.; Xu, G.; Shi, X. Q.; Van Hove, M. A.; Hsue, C.-S.; Tsuei, K.-D. Optimal Electron Doping of a $\mathbf{C}_{60}$ Monolayer on $\mathrm{Cu}(111)$ via Interface Reconstruction. Phys. Rev. Lett. 2010, 104, 036103. 


\section{Graphical TOC Entry}

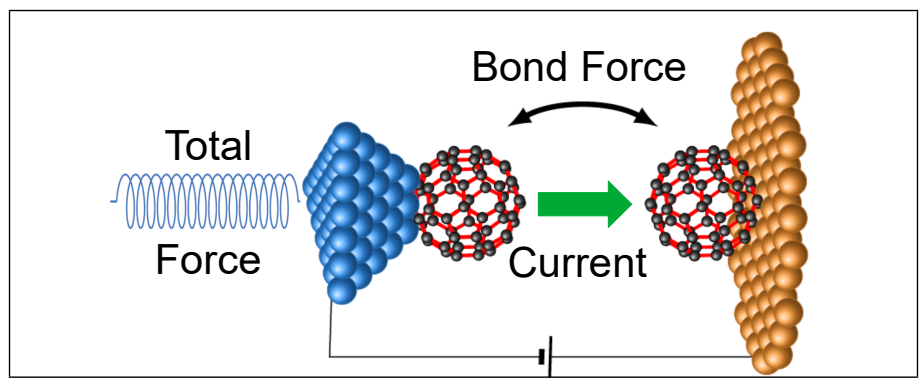

